\title{
Local Role of Food Producers' Communities for a Global One-Health Framework: The Experience of Translational Research in an Italian Dairy Chain
}

\author{
Chiara Frazzoli ${ }^{*}$, Alberto Mantovani1, Roberto Dragone ${ }^{2}$ \\ ${ }^{1}$ Department of Veterinary Public Health and Food Safety, Istituto Superiore di Sanità, Rome, Italy \\ ${ }^{2}$ Institute for Nanostructured Materials, Consiglio Nazionale delle Ricerche, Rome, Italy \\ Email: ${ }^{*}$ chiara.frazzoli@iss.it
}

Received January 2014

\begin{abstract}
Community is the foundation of public health: the present paper reports the approach and strategy for intervention on the dairy production community developed by the Italian project ALERT (www.alert2015.it), which implements the transfer of technical innovation and technological know-how from public research. Starting from the local role of primary producers, North-South and South-South networking (www.noodlesonlus.org) is needed to share solutions for transnational problems like climatic change, contaminated agro-farming sites and food waste and losses in the era of food crisis. Based on risk analysis, science-society dialogue and global health, the main drivers of this experience of translational research are One Health, i.e. the web of inter-relationships among environment, farm animals and human health, and sustainable food safety (prevention actions on diet of young women, today, to protect next generation's health) to increase population life expectancy in good health.
\end{abstract}

\section{Keywords}

Contaminated Sites; Ecology; Food Losses; Sustainable Food Safety; Technological Transfer; Traceability

\section{Introduction}

Translational research helps to make findings from basic science useful for practical applications that enhance human health and well-being; indeed, investment in health has been proven to boost development [1].

Protecting the food supply chain is an important component of health investment. In particular, prevention-based innovation in the dairy chain implies many facets at global level:

- Preserve quality and improve qualification and competitiveness of high-quality, territory-linked products and

${ }^{*}$ Corresponding author.

How to cite this paper: Frazzoli, C., Mantovani, A. and Dragone, R. (2014) Local Role of Food Producers' Communities for a Global One-Health Framework: The Experience of Translational Research in an Italian Dairy Chain. Journal of Agricultural Chemistry and Environment, 3, 14-19. http://dx.doi.org/10.4236/jacen.2014.32B003 
productions, e.g. Made in Italy cheeses (e.g. Parmigiano Reggiano, Grana Padano, Bufala Mozzarella Consortium). In this regard, the EXPO 2015 presents the Italian model of high quality products linked to territory while guaranteeing consumer safety.

- Improve accessibility of safe dairy products as nutritional source in developing countries [2]. Safe and nutritious food becomes all important since Non Communicable Diseases (NCDs) are projected to become the most common causes of death by 2030 in the developing world [3]; also, there is the need to support the citizens' trust in wholesome local food productions.

- Provide prevention highlights to the epochal change of East-Asian dietary habits now introducing milk.

- Support the change from production models highly impacting on environment to environmental sustainable models.

ALERT (www.alert2015.it) is a project funded by the Italian Ministry for Economic Development and based on the transfer of technical innovation and technological know-how emerged from public research. ALERT, coordinated by the Italian National Institute of Health (ISS), develops an innovative risk management framework and transferable technology to farm's daily production in order to exploit new biomarkers and new knowledge for early management of anomalies and production improvements (e.g. feed changes). The ALERT activities pivot on the bovine milk chain; nevertheless, parallel approaches and strategies might be developed also for milk chains of goats, buffalo, sheep, and under-utilized species in the dairy production, such as reindeer, elk, llamas, alpacas, donkeys, yaks, camels and mithun. Under this aspect, the shift of target consumers is very interesting and challenging; for instance: i) to date, safety aspects are better known for the bovine milk, whereas targeted approaches have still to be developed for many other dairy species; ii) animal milk is a major food especially for children aged 1 to 3 years [4], but consumption is customary in all age and gender groups, including women at fertile age which calls, therefore, for issues relevant to the next generation's health, i.e. sustainable food safety aspects [5]; iii) concerning the nutritional value, the under-utilized species present interesting aspects. The protein profiles of donkey and mare milk can make them better suited to the $2 \%-6 \%$ of the population allergic to bovine milk; in their turn, the milk of reindeer and moose contains less than half the lactose present in bovine milk and could provide a source alternately to the people who are lactose intolerant [2].

The above aspects should be viewed within the increasingly interconnected, globalized, food production and market system, including its "dark side", the global nutritional/food crisis and climatic change, that make the feed and food safety a emerged hot issue whose problems and solutions are transnational.

Farmers' communities increasingly stand out as societal components whose awareness rising and empowerment/endorsement are strategic to protect the health of populations. This is particularly challenging, due to the economic and environmental constraints that historically burden primary producers.

The Hazard Analysis and Critical Control Points (HACCP) approach is the widely used on-enterprise strategy to control and manage the safety of food production process as well as to support traceability and liability. The ALERT technological strategy, based on the bioelectronic multiprobe platform BEST patented by the ISS [6] implies the transfer of updated know how to innovate HACCP and self-monitoring plans with new biomarkers and chemical/toxicological aspects; the challenging milk matrix is both highly vulnerable to toxic contaminants and business core of a valuable as well as vulnerable group of food business operator like farmers [7].

Last but not least, milk is also interesting for its role as One Health (e.g. environmental quality) sentinel. ALERT characterises new transferable (i.e. automatically detectable on the farm through innovative marketable technology) biomarkers in a One Health perspective, since such biomarkers may indicate changes in animal health and/or husbandry and/or environment.

The driver of economy is food: the power and sovereignty of a land comes from within, from quality and safety of primary food productions.

\section{A Public-Private Initiative for Improved Daily Knowledge of Product and Production}

The European strategy for food safety [8] requires that the Official control, based on risk analysis "from farm to fork", is increasingly integrated by innovative systems for self-monitoring at each segment of the food chain (from primary production to transport, transformation, and marketing) by food business operators. According to the European strategy, food business operators are the subjects ethically and legally responsible for the safety of their own products. 
Between top-down food safety requirements and bottom-up responsibility of food operators is the role of public health scientists to provide innovation and know-how. In particular, effective two-lane (top-down and bottom-up) system for food safety requires the development of field biomarkers as automatically measurable biochemical or molecular indicators.

Basing on a multidisciplinary team (agronomists, veterinarians, biologists, chemists, engineers) the public-private Consortium of the project ALERT develops and transfers a automated technological platform based on (bio)detection tools (sensors and biosensors) and (bio)markers to the Italian dairy chain whose components are Pascolini (farm), Lattepiù (collection/transport), and Centrale del Latte di Roma/Parmalat (dairy industry).

ALERT responds to the demand of dairy producers for optimizing the use of resources devoted to self-control by adopting technologies for early warning of safety, quality and nutritional factors of their products. When implemented, the new strategy will decrease the vulnerability to unexpected events, reduce the risk of product recalls from the market and the related costs of food destruction as well as damage to the enterprise's commercial image.

The mentioned food business operators of the dairy chain started the ALERT Consortium with a cluster of public institutes with top-level expertise in food chain protection (ISS, IZSLT) and technological transfer (CNR), and a cluster of enterprises (Amel, Biosensor, TDM) with established expertise in sensing systems. The Consortium management aspects are dealt with separately by another enterprise (LBC).

\subsection{Technological Transfer}

The BEST platform is conceived and designed to support in continuum control and management of wholesomeness, quality, safety and traceability of the bovine milk chain, from farm through to transport, treatment and distribution. BEST is an integrated array of sensors and biosensors working in parallel. The hardware platform is based on up to 40 automatic real-time in-situ chemical and biological probes, simultaneously measuring chemical-physical ( $\mathrm{pH}$, conductivity, temperature, redox potential), specific (glucides, urea, oxidative stress, heavy metal- and pesticide-related enzyme changes) and biological (cell respiration and catabolism, bacterial burden) parameters.

Probes are currently based on electrochemical and optical sensors, and on enzymes and whole cells as biological media in biosensors. Thanks to remote (Wi-Fi) technical control and data assessment, product and production are both daily monitored and chances for traceability along the whole chain are set up.

ALERT exploits the technologies developed so far in the field of sensors and biosensors towards recognised official application in food safety:

- The so-called "weakness" of biosensors, i.e. qualitative or semi-quantitative output generally used for screening purposes only, is exploited for self-targeted monitoring purposes thanks to control charting.

- The selection of qualitative, semi-quantitave or presence/absence output depends on the nature and aim of the investigated parameters.

- The limit of detection of available probes currently considered useless become suitable for non-regulated parameters (e.g. total exposure biomarkers).

- Up-to-date techniques of the microelectronics and microfluidics industry are exploited for assembling chemical-physical sensors as biosensors' transducers also.

- Novelty in both recognition and transduction process for realistic analytical measurements during daily food production; e.g. engineered receptors for recognition of specific molecules are exploited.

(Bio)sensors arrays have the potential to become widely accepted for self-diagnostic and self-monitoring applications, provided that robust results on fully automated platforms are successfully generated. For this purpose:

- BEST is an open and flexible technology able to improve its detection capability by hosting new probes made available by the scientific community.

- Automation is exploited for easiness of use and maintenance, including minimization of reagents and costs, robustness to field conditions and acoustic/light signaling, thus facilitating acquisition/readability by the end-users.

\subsection{Transfer to Risk Management}

For effective transfer, the core principle of ALERT is to support the bottom-up role of producers in guaranteeing 
food safety, thus reducing and refining downstream external control on final products. ALERT will improve self-monitoring capability and facilitate its acquisition by adopting the end-users perspective: control charting (already recognized and adopted in HACCP systems), integration with previous enterprise's database, and HACCP-like monitoring of anomalies respect to the general trend in the specific enterprise. The ALERT conceptual framework highlights invariability as a quality parameter: expected values are self-targeted, set on the enterprise historical characteristics rather than established from external bodies.

ALERT offers an analytical criterion to integrate the current statistical-based, randomized criterion foreseen by official control schemes and self-monitoring plans, thus optimizing the use of resources devoted to official control. Moreover, the official food safety system will benefit from food operators empowered in their knowledge of the food production chain. To facilitate interface and recognition by official bodies, the BEST-based self-monitoring performances are assessed along with data from official methods and certified/reference materials.

Basing on acceptance by both food operators and official bodies, ALERT develops the following:

- Designs modern HACCP- and good practices-based self-monitoring plans including the toxicological risks [7] to support decision making in points of particular attention of the production line.

- Identifies and characterizes innovative (grids of) (bio)markers of undesirable (chemical and microbial) pollutants, milk quality (composition, subclinical mastitis, and metabolomics) and anomalies in bioactive concentrations (e.g. net antioxidant power impacting on oxidative shelf life), facing interpretation challenges such as dose-response(s) relationships and matrix effect. The core idea is that $n$ clues make a test.

- Investigates the whole food approach based on signals from the bulk mixtures of molecules present in the same commodity and having common mode of action or biological target, rather than the same structure.

- Develops guidelines for recognition by the official control with the strategic aim of official acceptance within the national and international food safety systems.

Finally, ALERT fosters the One Health framework in food safety, as its successful outcome will support the investigation of:

- One Health biomarkers in farm animals for daily monitoring by food producers including both targeted (e.g. heavy metals, micotoxins, pesticides, veterinary drugs) and total exposure (e.g. oxidative stress or cells respiration related) biomarkers.

- Detoxification of farm animals in contaminated sites.

- Impact of different production practices (intensive, traditional, organic, and biodynamic).

Last but not least, through the follow-up of biomarkers ALERT seeks to establish traceability along the whole chain.

\subsection{Transfer to the Market}

Marketability, i.e. effective need, sustainability and impact, of the ISS Spin-off proposal named MILKNET has been analysed in the frame of business plan competitions. The MILKNET entrepreneurial project won the Start-Cup CNR-IlSole24Ore Prize for Central Italy in 2011; MILKNET was one of the 3 finalists (out of 560 projects competing) at the 2011 edition of the National Innovation Prize-Working Capital (Social Innovation area) [9].

The drivers for marketability lie in the legal responsibility of food operators, the role of consortia and associations, the adherence to widely recognised norms, the increasing demand for safety by the consumer, as well as in the measures to facilitate diffusion in farming enterprises.

In Countries where official control systems are strong, MILKNET offers innovation of both product (qualification and competitiveness) and process. In developing and emerging Countries, which will gain a greater role in the global food market, MILKNET supports the implementation of the web of bottom-up prevention actions as driver of change.

\section{Sharing Solutions for Transnational Problems}

\subsection{The Community Participatory Approach}

Community is the foundation of public health and identifying communities is the first step in facilitating community change. The community context can be exploited to promote and encourage behaviour changes in public 
health, provided that community components, structure, characteristics (e.g. norms, constraints, capacities and resources), typologies (e.g. farmers, consumers, citizens, and reasons for grouping) as well as awareness and attitudes towards empowerment are correctly identified and understood [10]. Involvement deeply affects sustainability of community change efforts: communities need to participate in the design, implementation and evaluation of any intervention. Provided proper resources and empowerment (e.g. know how and technology), the proactive role of farmers is strategic to integrate top-down public health measures under multiple aspects, like:

- One Health, including environmental health (e.g. animal farm waste) and innovation (e.g. assessment of deliberate changes in the production process, e.g. animal diet, self-produced or acquired feeds, watering source).

- Rural development, including efficacy, diffusion and effectiveness of the BEST-based approach for sharing information and intervention measures (e.g. telediagnostics).

- Climate change and seasonal effects, including geoclimatic factors, e.g. changes in the dynamics of pollutants and nutritional composition of soil and vegetables.

- Emergencies, including early identification of commercial frauds (e.g. water dilution of milk or mixing with milk from different species) possibly hindering safety issues, or unintended/unexpected contaminations.

- Emerging or re-emerging problems, possibly highlighted by the monitoring of arrays of signals.

- Contaminated agro-zootechnic sites, waste burning or by-products contaminations [11], including follow-up of remediation activities.

- Food waste and losses, including exploitation of surplus, scrap, and agro-food by-products.

\subsection{The Web Open Platform}

The BEST bioelectronic platform is designed as an open flexible technology, which is capable of updating through the integration of new probes and/or biomarkers.

In order to survey the probes developed as field transferable tools by the scientific community (universities, research institutes, companies, etc), the ALERT consortium has launched a census web tool

(http://www.alert2015.it/census). On the other side, the BEST technology meets emerging issues posed by its end-users (farmers' associations, consortia, dairy industry) through a similar web database

(http://www.alert2015.it/issues). To match issues and probes, knowledge in risk assessment is supported in ALERT by in silico tool (QSAR) with the final objective of improving the BEST's diagnostic capabilities.

Based on this approach, ALERT establishes a platform for long-term interface between dairy enterprises and research bodies to facilitate science-society dialogue and fostering effective and updated innovation.

\subsection{Global Health}

The ALERT initiative is amplified by the NOODLES Network (Nutrition \& food safety and wholesomenessPrevention, education and research, www.noodlesonlus.org) to boost science-food producers dialogue and foster the Global Health framework by working locally but establishing North-South and South-South collaboration (currently in Mid East, Asia and sub-Saharan Africa) around the ALERT objectives.

The global strategy on NCDs presents four key tensions [3]:

- The tension between human rights and corporate rights, including the right to informed choices, and of freedom from unintended exposure. This tension calls for long-term vision, and development of science-driven healthcare based on primary prevention in face to the business- and technology-driven healthcare based on costly treatments and drugs.

- The tensions regarding where to invest in health along the "causal chain" (from social determinants to treatment services), including tackling underlying causes of the diseases (prevention) or to invest on care for those already ill.

- The tensions in governments funding programs for their own countries versus assistance for developing countries, including the chance of re-allocating existing funds to prevent/reduce the growing burden of NCDs or shifting from disease-specific programmes to health systems strengthening.

- The tensions about which intervention to prioritize: focus on people who most need, on people who would benefit most, on actions that would benefit the most people due to common causes. Dealing with such tensions calls for risk analysis, which has to be driven by community values and supported by scientific data. 


\section{Acknowledgements}

The authors acknowledge the Consortium of the project ALERT (www.alert2015.it) "Integrated system of biosensors and sensors ("BEST") for the monitoring of wholesomeness and quality, as well as for traceability in the cow milk chain” funded by the Italian Ministry of economical development under the Call Industria 2015 "New technologies for Made in Italy” and the team of the NOODLES Network (www.noodlesonlus.org).

\section{References}

[1] Clift, J., Ed. (2004) Health and Development. International Monetary Fund, Washington DC, USA.

[2] Muehlhoff, E., Bennett, A. and McMahon, D., Eds. (2013) Milk and Dairy Products in Human Nutrition. Food and Agriculture Organization of the United Nations (FAO), Rome.

[3] Venkatapuram, S., McKee, M. and Stuckler, D. (2012) Ethical Tensions in Dealing with Noncommunicable Diseases Globally. Bulletin of the World Health Organization, 90, 241-242.

[4] European Food Safety Authority (2009) Panel on Dietetic Products Nutrition and Allergies. Scientific Opinion on the Appropriate Age for Introduction of Complimentary Feeding of Infants. EFSA Journal, 7, 1423. http://dx.doi.org/10.2903/j.efsa.2009.1423

[5] Frazzoli, C., Petrini, C. and Mantovani, A. (2009) Sustainable Development and Next Generation's Health: A LongTerm Perspective about the Consequences of Today's Activities for Food Safety. Annali dell'Istituto Superiore di Sanità, 45, 65-75.

[6] Frazzoli, C., Mantovani, A., Campanella, L. and Dragone R. (2010) Technological Integrated Bioelectronic System and Relevant Control Charting for Early Intervention on Food Chain and the Environment [BEST]. PCT Patent WO 2010/001432 A1.

[7] Frazzoli, C. and Mantovani, A. (2010) Toxicants Exposures as Novel Zoonoses: Reflections on Sustainable Development, Food Safety and Veterinary Public Health. Zoonoses and Public Health, 57, e136-e142. http://dx.doi.org/10.1111/j.1863-2378.2009.01309

[8] Commission of the European Communities (2000) White Paper on Food Safety, COM/99/0719 Final—Not published in the Official Journal.

[9] Frazzoli, C., Mantovani, A. and Dragone, R. (2012) The ISS Patent “BEST” for Timely Risk Management in Veterinary Public Health and Food Safety: Public Research Meets Enterprise) [ITA]. Notiziario dell'Istituto Superiore di Sanità, 25.

[10] Frazzoli, C. (2012) Trade Not Aid: Challenges for Market Drivers of Safe Foods in Africa. In: Frazzoli, C., Asongalem, E.A. and Orisakwe, O.E., Eds., Cameroon-Nigeria-Italy Scientific Cooperation: Veterinary Public Health and Sustainable Food Safety to Promote “One Health/One Prevention”. Rapporti ISTISAN 12/49, Istituto Superiore di Sanità, Rome, 60-69.

[11] Frazzoli, C., Orisakwe, O.E., Dragone, R. and Mantovani, A. (2010) Diagnostic Health Risk Assessment of e-Waste on the General Population in Developing Countries' Scenarios. Environmental Impact Assessment Review, 30, 388-399. http://dx.doi.org/10.1016/j.eiar.2009.12.004 